Revue

de I'histoire des religions

\section{Revue de l'histoire des religions}

$4 \mid 2005$

Lieux de culte, lieux saints dans le judaïsme, le christianisme et l'islam

\title{
« Lieux juifs » : solitude du Mont, rumeurs du monde
}

"Jewish Places": The Isolated Mount, world rumours

\section{Régine Azria}

\section{(2) OpenEdition}

\section{Journals}

Édition électronique

URL : http://journals.openedition.org/rhr/4230

DOI : $10.4000 /$ rhr.4230

ISSN : 2105-2573

Éditeur

Armand Colin

Édition imprimée

Date de publication : 1 octobre 2005

Pagination : $557-572$

ISBN : 2200-92087-3

ISSN : 0035-1423

Référence électronique

Régine Azria, « "Lieux juifs » : solitude du Mont, rumeurs du monde », Revue de l'histoire des religions

[En ligne], 4 | 2005, mis en ligne le 18 janvier 2010, consulté le 20 avril 2019. URL : http://

journals.openedition.org/rhr/4230 ; DOI : 10.4000/rhr.4230 


\section{« Lieux juifs » : solitude du Mont, rumeurs du monde}

Le génie bâtisseur que les juifs se sont redécouverts à la faveur de l'établissement d'un État juif ne s'est pas investi dans des projets religieux mais dans des édifices à vocation profane: la plupart des synagogues d'Israël sont discrètes et se fondent dans le tissu urbain. Elles s'inscrivent dans la tradition d'une longue expérience de familiarité avec les choses du sacré qui n'a pas grand-chose à voir avec l'expérience du face-à-face solitaire avec le divin d'un Mö̈se ou d'un Abraham, ni même avec l'expérience vécue par les juifs lors des pèlerinages d'antan au Temple de Jérusalem. Mais les juifs diasporiques se reconnaissent aujourd'hui dans d'autres lieux emblématiques, tels que les musées et les lieux de mémoire.

\section{"Jewish Places": The Isolated Mount, world rumours}

The building spirit that Jews have rediscovered thanks to the foundation of a Jewish state, did not materialize in religious but in profane buildings: most Israeli synagogues are inconspicuous and merge into the urban texture. They are inscribed in a long traditional experience of familiarity with things sacred, and have not much to do with Moses' or Abraham's solitary face to face with deity, nor even with what Jews experienced during past years pilgrimages to Jerusalem Temple. But today diaspora Jews find their bearings in other emblematic places such as museums and remembrance places. 
Malgré sa longue histoire, le judaïsme n'a jusqu'à ce jour rien versé au patrimoine architectural religieux de l'humanité qui puisse rivaliser avec la splendeur des églises d'Occident, le raffinement des mosquées d'Orient ou des temples d'Extrême-Orient. Le seul monument juif qui eût pu prétendre entrer dans la compétition, et dont les juifs auraient éventuellement pu s'enorgueillir, n'existe plus. C'était le Temple de Jérusalem, ce Temple magnifique et solitaire dressé sur le Mont du Rocher, sur le lieu même où, d'après la tradition juive, Abraham s'apprêtait à immoler son fils Isaac, ce Temple à propos duquel Flavius Josèphe n'avait pas de mots assez forts pour en décrire la beauté. Manque de chance, par deux fois, ce Temple a été détruit (en $586 \mathrm{av}$. J.-C. et en 70 de notre ère), et depuis près de deux mille ans, aucun monument comparable, par la splendeur, la dimension, ou la fonction, n'est venu le remplacer.

Il est intéressant d'observer que la reconstruction d'un Temple à Jérusalem n'a, à aucun moment, figuré parmi les priorités des juifs à l'époque moderne, ni lors de la création de l'État d'Israël ni dans les décennies qui ont suivi. Depuis le retour des juifs à Jérusalem au début du $\mathrm{XX}^{\mathrm{e}}$ siècle, ni les responsables politiques ni les autorités rabbiniques du pays ne l'ont envisagé, même après la reconquête des lieux saints par l'armée israélienne en 1967. Les penseurs du sionisme moderne ne semblent pas avoir prévu, eux non plus, de rubrique "construction du troisième Temple » à leur programme. Ce qui ne veut pas dire que personne n'y ait songé ou n'ait été tenté par l'entreprise. Car les militants du $3^{\mathrm{e}}$ Temple existent. Ils sont peu nombreux, certes, mais déterminés. Ils sont actifs et ils disposent de moyens et de relais. Pour preuve, ils ont ouvert une yechiva et un institut de recherche dans la vieille ville de Jérusalem, à deux pas du Har haBaït, le Mont du Temple. Qui plus est, ils savent périodiquement attirer l'attention des médias et donner des sueurs froides aux forces de sécurité israéliennes grâce à des actions d'éclat : par exemple, lorsqu'ils viennent déposer ce qu'ils prétendent être «la première pierre du troisième Temple » sur l'esplanade des Mosquées, un lieu pourtant étroitement surveillé. Ces activistes qui font des émules et qui ne peuvent plus être considérés comme de simples marginaux, sont tout sauf de doux rêveurs. Ils ne se contentent pas 
d'être des mystiques un peu trop exaltés. Ce sont de redoutables illuminés, des militants prêts à tout, et qui font peser une menace réelle sur la paix et la stabilité de la région. C'est là une des raisons pour lesquelles les autorités d'Israël veillent à prévenir toute initiative de leur part qui s'apparenterait à une ébauche de mise en œuvre de leur projet messianique de reconstruction. L'ampleur des conséquences de l'ouverture d'un tel chantier est difficile à imaginer, lorsqu'on sait que la construction du troisième Temple de Jérusalem suppose un préalable, à savoir la destruction de deux édifices qui comptent parmi les hauts lieux de l'islam : le Dôme du Rocher, connue sous le nom de Mosquée d'Omar, et la mosquée Al Aqsa. Pourquoi les détruire ? Parce que ces mosquées se dressent précisément sur le lieu présumé des deux temples détruits, c'est-à-dire à l'endroit même où, s'il venait à être construit, devrait être érigé le troisième du nom. Autrement dit, malgré le retour des juifs sur la terre de leurs lointains ancêtres, le scénario post-babylonien du second Temple ne semble pas devoir se reproduire une seconde fois..., du moins peut-on l'espérer.

Cela ne signifie pas que l'État d'Israël et ses représentants soient insensibles à la dimension architecturale, voire monumentale, de la renaissance nationale juive. Ils n'ignorent pas que l'idée de la renaissance d'un État juif sur le territoire de la Bible n'est pas le fait du hasard. Ils savent que l'utopie à l'origine du sionisme politique, celle qui visait à renouer concrètement le lien avec le mythe et l'histoire hébraïques, trouve sa source, en dépit de son caractère nonreligieux, voire antireligieux, dans la tradition prophétique juive, dans un imaginaire saturé de représentations religieuses et de formules qui, toutes, font référence au retour à Sion et à Jérusalem : « si je t'oublie, Jérusalem... », «l'an prochain à Jérusalem », et pas à n'importe quelle Jérusalem, à la Jérusalem reconstruite dans ses murailles autour de son Temple. Et pourtant ! Pour puissant, sacralisé et aussi fidèlement transmis de génération en génération qu'il ait pu être, l'impact de ces références et de cette mémoire n'a pas suffi. Ce qu'on observe lorsqu' on circule aujourd'hui en Israël c'est précisément l'inverse : l'absence de toute "présence monumentale du divin ». Les grandes réalisations architecturales à caractère 
monumental qui marquent avec le plus de force la renaissance d'un État juif ne se présentent ni sous la forme d'un hypothétique troisième Temple, ni même sous celles de synagogues dont les qualités architecturales seraient propres à susciter l'émerveillement. Jusqu'à ce jour, les réalisations les plus spectaculaires ont été et continuent à être des ouvrages à caractère profane, quelles que soient leurs fonctions, que celle-ci soit politique, avec la Knesset ; juridique, avec la Cour suprême qui fait face à la Knesset ; administrative, avec la très moderne Municipalité de Jérusalem ou le tout nouveau Ministère des Affaires étrangères (inauguré en 2003) ; que ces bâtiments aient une vocation académique et scientifique (universités, fondations, instituts de recherche), une vocation culturelle (musées, théâtres, auditorium), une vocation mémorielle (le mémorial de la déportation de Yad Vashem et les nombreux monuments à la mémoire des soldats morts pour la patrie qui parsèment le pays). Cette liste n'est évidemment pas exhaustive.

Cette énumération entend signifier que le génie bâtisseur et le souci esthétique que les juifs se sont redécouverts à la faveur de l'établissement d'un État juif, ne se sont pas investis spontanément ou prioritairement dans des projets cultuels ou religieux. Ce qui ne veut pas dire qu'Israël soit dépourvu d'édifices religieux présentant un intérêt architectural. De belles synagogues, esthétiquement innovantes, ont elles aussi vu le jour dans la foulée : celle de l'hôpital Hadassah à Jérusalem, construite pour accueillir les vitraux de Chagall, celle de l'Université hébraïque, également intéressante. Mais aucune ne peut prétendre rivaliser avec les édifices chrétiens ou musulmans. Pourtant, les synagogues ne manquent pas en Israël. Il y en a beaucoup, des milliers. Mais la plupart sont discrètes; elles se fondent dans le tissu urbain et passent le plus souvent inaperçues. On ne les remarque pas ou on n'y prête tout simplement pas attention. Leur présence dans le paysage apparaît naturelle et normale. C'est davantage par l'oreille, grâce aux sons qui traversent leurs murs ou s'échappent des fenêtres qu'on les repère. Le bruissement d'une synagogue ne se confond avec aucun autre bruit, aucune autre musique. Il est immédiatement identifiable par quiconque l'a entendu une fois. La plupart de ces synagogues sont de taille modeste, très 
modeste même. Et si on ne les remarque pas, c'est aussi et surtout parce que beaucoup sont de simples oratoires, de petites salles de prières aménagées de façon rudimentaire, installées au rez-dechaussée d'immeubles ordinaires. Elles sont fréquentées par des habitués, qui n'ont besoin ni de decorum ni de fastes et qui se satisfont du minimum nécessaire pour prier et étudier leur page de Talmud quotidienne ou hebdomadaire : des chaises, des bancs, des tables, des rayonnages pour les livres, un pupitre pour l'officiant, une armoire pour les rouleaux de la Torah, un lavabo pour les ablutions rituelles, leur suffisent. En général on se retrouve dans ces synagogues de quartier entre habitués parce qu'on est de même origine, qu'on vient du même pays ou de la même ville, parce qu'on prie dans le même rite avec le même accent, parce qu'on appartient à la même obédience religieuse et qu'on vénère le même rabbin, ou plus simplement encore pour des raisons de commodité, parce qu'on habite à côté.

Ces « lieux » participent d'un mode de vie encore très actuel dans certains milieux juifs, en Israël comme en diaspora. La synagogue y trouve naturellement sa place entre l'épicerie et le jardin d'enfants. Au cœur de la rumeur du monde, elle a sa place dans la vie de tous les jours. Tels qu'ils se présentent, dans leur simplicité et leur dépouillement, ces lieux de prière et d'étude ne suscitent pas d'étonnement. Ils appartiennent au paysage banal de la rue juive et s'inscrivent dans la continuité de la tradition, celle d'une longue expérience de familiarité avec les choses du sacré, d'un sacré proche qui n'a pas grand-chose à voir avec l'expérience d'un Moïse ou d'un Abraham et de leur face à face solitaire avec le divin, ni même avec l'expérience du sacré telle qu'elle devait être vécue par les juifs lors des pèlerinages au Temple de Jérusalem, mais d'un sacré de tous les jours, fait d'habitudes et de gestes ordinaires, comme le fait d'observer les règles de la cacherout, d'embrasser la mezouza en pénétrant dans une maison ou de se reposer le chabbat, d'un sacré banalisé, sans faste ni apparat, intégré à la vie et qu'on n'aurait pas l'idée d'isoler ou de séparer du reste.

Qu'en est-il, maintenant, des lieux de prière juifs hors d'Israël ? À l'évidence, la précarité relative de la condition diasporique et 
minoritaire n'a pas prédisposé les juifs à devenir des experts en architecture du divin. L'histoire ne leur a pas offert les mêmes possibilités que celles qu'ont connues le christianisme et l'islam pour inscrire durablement et de façon aussi souveraine, la trace de leur présence dans l'espace et dans le sol. Eux-mêmes, d'ailleurs, pendant longtemps, n'ont pas compté ce souci parmi leurs priorités. Non pas parce qu'ils aimaient moins leur dieu que les chrétiens, mais peut-être plus simplement, comme je viens d'essayer de le montrer, parce qu'ils l'aimaient autrement, à travers des gestes et des rites. Ce qui a pu inspirer au théologien judéo-américain, Abraham Heschel, l'invention de la belle expression de «bâtisseurs du temps » pour qualifier les juifs ${ }^{1}$. À la différence d'autres peuples d'autres religions, les juifs auraient été des «bâtisseurs du temps » plutôt que des bâtisseurs de temples ou de monuments.

\section{LA SYNAgogue, L'ÉGLISE, LE TEMPLE...}

Pourtant, et indépendamment des circonstances, cette supposée indifférence pour la pierre ou le bâti n'a jamais été absolue chez les juifs et elle a connu de notables exceptions. Car les juifs ont été des bâtisseurs d'édifices religieux sur les lieux de leur dispersion, dont certains fort beaux et qui figurent en bonne place dans le patrimoine de l'humanité : de Doura Europos à Prague, des synagogues de l'Espagne andalouse à celles de Pologne ou de Transylvanie. Il existe de nombreux travaux sur la question, aussi ne m'y attarderai-je pas ${ }^{2}$.

1. Abraham Heschel, Les bâtisseurs du temps, Paris, Éditions de Minuit, 1957.

2. Joseph Gutmann, The Synagogue: Studies in Origins, Archaeology and Architecture, New York, Ktav Pub. House, 1975 ; Dominique Jarrasse, L'Âge d'or des synagogues, Paris, Herscher, 1991 ; Carol Herselle Krinsky, Synagogues of Europe: architecture, history, meaning, Cambridge (Mass.), The Architectural History Foundation, MIT Press, 1985 ; Maria et Kazimierz Piechotka, Wooden Synagogues, Varsovie, 1959 ; Rahel Wischnitzer-Bernstein, The Architecture of the European Synagogue, Philadelphie, The Jewish Publication Society of America, 1964. Par ailleurs, dans l'ouvrage monumental de Gabrielle Sed-Rejna (L'art juif, Paris, Citadelles et Mazenod, 1995), une importante bibliographie figure en fin d'ouvrage. 
Mais, plus encore et surtout, nous sommes fondés à nous demander si cette belle formule de "bâtisseurs du temps », reste encore d'actualité aujourd'hui. C'est pourquoi je serais tentée de poser les questions suivantes :

1) Existe-t-il, aujourd'hui, des « lieux » ou des formes d'inscription spatiale dans lesquels les juifs se reconnaissent plus que d'autres? Si oui, quels sont-ils ?

2) Quelle est, aujourd'hui, la place de la synagogue dans la vie réelle des juifs et dans les représentations?

3) Cette seconde question en entraîne une autre : quel « lieu » la synagogue est-elle, comparée à l'église ? Et qu'en est-il du Temple?

Deux niveaux de comparaison donc, l'un externe et l'autre interne au judaïsme, pour tenter de comprendre ce qu'est et ce que n'est pas la synagogue, pour tenter de comprendre aussi les raisons de sa centralité en même temps que celles de sa relative marginalisation et de son remplacement, à l'époque moderne et contemporaine, par de nouveaux lieux emblématiques.

C'est à partir d'un constat inquiet : celui de la désaffection religieuse des catholiques, que le doyen Le Bras d'abord, le chanoine Boulard ensuite, ont posé les bases de ce qui allait devenir en France la sociologie de la religion ${ }^{3}$. Sur quoi ce constat se fondait-il ? Sur le fait que les églises se vidaient, qu'elles étaient de moins en moins fréquentées par les fidèles et que cette désaffection n'était pas uniforme : elle avait sa géographie, sa morphologie sociale, etc. C'est ainsi que la grande enquête sur la pratique religieuse des catholiques français de Gabriel Le Bras, tout comme la géographie de la France religieuse de Fernand Boulard allaient l'une et l'autre

3. Fernand Boulard, «Carte de la pratique religieuse de la France rurale », dans Cahiers du clergé rural, 1947 ; Problèmes missionnaires de la France rurale, 2 vol., Paris, Le Cerf, 1945 ; Premiers itinéraires en sociologie religieuse, Paris, Éditions ouvrières/Économie et Humanisme, 1954 (préf. de Gabriel Le Bras); Gabriel Le Bras, Études de sociologie religieuse, Paris, PUF, 1955-1956 ; Fernand Boulard, Jean Rémy, Pratique religieuse urbaine et régions culturelles, Paris, Éditions Ouvrières/Économie et Humanisme, 1968 ; François-André Isambert, Jean-Paul Terrenoire, Atlas de la pratique religieuse des catholiques en France, Paris, Fondation Nationale des Sciences Politiques/CNRS, 1980 (établi sur la base des matériaux laissés par le chanoine Boulard). 
s'élaborer sur ce socle dur, qui apparaissait alors comme l'indicateur par excellence de la religiosité catholique et de la pratique religieuse : l'assistance à la messe. La mesure de la fréquentation de l'église allait constituer un des axes majeurs de la sociologie du catholicisme, la classification des fidèles, opérée par Boulard en 1931, établissant la distinction entre les « détachés », les « conformistes saisonniers » les « pratiquants réguliers » les « fidèles dévots ou zélés».

Peut-on imaginer démarche du même ordre en terrain juif, c'està-dire imaginer une sociologie qui retiendrait comme principal indicateur de la religiosité juive la fréquentation de la synagogue : fréquentation quotidienne, hebdomadaire (le dimanche se voyant remplacé par le chabbat), festive, occasionnelle ou liée aux rites de passage ? Posée en ces termes, la question prête à sourire dans la mesure où chacun sait qu'une grande partie des pratiques religieuses juives sont des rites domestiques, qu'on rencontre hors de la synagogue.

Elle laisse perplexe parce qu'une démarche de ce type ne trouverait sa justification qu'à une condition : considérer comme fondé le postulat de l'équivalence fonctionnelle entre l'église et la synagogue. Plus en amont encore, pour être épistémologiquement fondée, cette démarche devrait faire le pari d'une identité structurelle entre le fait religieux juif et le fait chrétien. Selon ce postulat ou ce pari, aller à la synagogue serait alors l'équivalent pour un juif du fait d'aller à l'église pour un catholique. Ce qui n'est évidemment pas le cas. Ni le postulat de l'équivalence fonctionnelle ni le pari de l'identité structurelle ne tiennent: théologiquement et sociologiquement, judaïsme et christianisme n'accordent pas la même signification au fait d'aller régulièrement à la synagogue et au fait de fréquenter assidûment l'église. Car, de même que le rabbin n'est pas un curé juif, ni même un prêtre dans le sens classique du terme, de même l'église et la synagogue ne sont pas l'équivalent l'une de l'autre. Ce qui ne veut pas dire que la synagogue soit sans intérêt pour notre réflexion. Bien au contraire. De fait, elle occupe une place importante, voire centrale à certains égards. Mais encore fautil se demander laquelle et de quelle façon. Pour répondre il nous faut revenir à une histoire des représentations. 
Dans le monde chrétien, et plus particulièrement catholique, la vision théologique préconciliaire qui a longtemps prévalu s'est attachée précisément à poser l'équivalence Synagogue/Église (ecclesia), le plus souvent aux fins de disqualifier la première. Cette équivalence est particulièrement frappante et édifiante dans l'art médiéval et ses représentations visuelles du face-à-face entre l'Ancienne et la Nouvelle Alliance, entre un judaïsme politiquement et spirituellement vaincu, c'est-à-dire expulsé hors de l'Histoire et dépossédé de son élection, et une chrétienté victorieuse, dominatrice et sûre d'ellemême. Je pense, entre autres, à la cathédrale de Strasbourg où, depuis le flanc d'un de ses portails latéraux, la figure allégorique de l'Église triomphante représentée sous les traits d'une belle femme au port altier, n'en finit pas, depuis le Moyen Âge, de défier une Synagogue aux yeux bandés, représentée sous les traits d'une femme jeune et belle, certes, mais qui n'en porte pas moins les signes visibles de sa défaite et de son humiliation ${ }^{4}$. Dans cette représentation chargée de symboles, la Synagogue ne désigne évidemment pas la maison de prière des juifs. Elle figure au titre d'équivalent structurel, par analogie avec la polysémie du mot «église», avec ou sans É majuscule - à la fois édifice et institution - comme le symbole du judaïsme compris en tant qu'institution, qui plus est, en tant qu'institution déchue de son Élection et de sa vocation universelle : être une lumière pour les nations !...

Et de fait, cette signification où la partie désigne le tout correspond aussi à ce qui a longtemps été historiquement et socialement vécu comme une réalité : celle d'une synagogue à la fois institution centrale des juifs, maison de prière, d'étude et de réunion, sorte de Parlement local des juifs de diaspora, comme son nom, beit knesset, le suggère. Car la synagogue est une très vieille institution. À en croire l'Encyclopaedia Judaica, c'est elle qui aurait servi de modèle aux chrétiens et aux musulmans en matière

4. On trouve la même représentation allégorique dans les niches extérieures de la cathédrale de Bamberg en Allemagne (fin du XIII ${ }^{\mathrm{e}}$ siècle), à Reims, Paris, à l'église St Séverin de Bordeaux, dans la cathédrale de Lincoln... Voir Bernhard Blumenkranz, Le juif médiéval au miroir de l'art chrétien, Paris, Études Augustiniennes, 1966, p. 105 sq. 
d'organisation du culte public ${ }^{5}$. Bien que son origine exacte soit mal établie, on la sait antérieure à la destruction du second Temple. Ce que confirme l'ouvrage de référence que constitue la somme de Lee Levine, The Ancient Synagogue 6 . Mais c'est en diaspora qu'elle devient une institution à part entière. On peut supposer que ses origines remontent à la période de l'exil babylonien : privés de Temple, établis dans un pays étranger, les exilés auraient éprouvé la nécessité d'avoir des lieux pour s'y réunir. Il semble logique que le besoin de se rassembler pour prier ou se ressourcer ait été ressenti de façon plus forte en diaspora qu'en Palestine où la présence du Temple captait l'essentiel de la participation religieuse des fidèles en même temps qu'elle confortait le sentiment d'unité et de cohésion du peuple. Mieux protégés des pressions externes, les juifs palestiniens avaient moins besoin de la synagogue que ceux de diaspora pour y affirmer collectivement leur identité. Avec la destruction du Temple en 70, c'est donc la synagogue qui prend le relais. Moyennant de sévères révisions visant à transformer la religion mère, en l'occurrence une religion archaïque attachée à un territoire et à un culte sacrificiel, en une religion attachée au rite, à la prière, à l'étude et à l'observance de la Loi, la synagogue devient l'institution centrale des juifs, l'espace à partir et autour duquel ils organisent leur vie religieuse et sociale, le leadership communautaire étant confié à des notables laïcs et le leadership religieux à une nouvelle élite, le corps rabbinique, gardien et gestionnaire de la Loi. Ainsi, à la différence du Temple, cette bâtisse unique, imposante et vide, interdite aux simples fidèles, la synagogue, démultipliée en autant de fragments que la dispersion, est la maison des fidèles. Elle le restera, aussi longtemps que le système communautaire saura résister aux effets dissolvants de la modernité et de la sécularisation.

Sauf lorsqu'elle est évoquée en tant que figure allégorique, la synagogue ne saurait donc être écrite avec un S majuscule, comme l'Église l'est avec un É majuscule, l'usage de la majuscule suggérant,

5. Encyclopaedia Judaica, XV, Jérusalem, The McMillan Company, 1971, p. 579.

6. Lee Levine, The Ancient Synagogue. The First Thousand Years, Yale University Press, 2000. 
selon nos conventions typographiques, une forme d'absolutisation. Or, la synagogue est étrangère à toute forme d'absolutisation. C'est une institution humaine où règnent la ferveur, le désordre et le bruit. C'est probablement à la révélation bouleversante que constitua pour lui la découverte de cette ferveur populaire, bruyante et d'un autre âge, que le philosophe juif allemand Franz Rosenzweig doit de n'avoir pas franchi le pas de la conversion ${ }^{7}$. La synagogue est étrangère à toute forme de mise en majuscule, sauf à l'époque moderne, lorsqu'elle commence à s'assimiler et qu'elle cherche précisément à imiter le modèle chrétien. Dès lors en effet, elle devient grande, claire, monumentale, ordonnée, solennelle; policée, elle adopte l'orgue et le chœur pendant que ses administrateurs revêtent la jaquette et le haut de forme. Mais, pour beaucoup de juifs attachés à leur antique tradition, cette synagogue n'est plus vraiment une synagogue. D'ailleurs on ne l'appelle plus ainsi. Pendant que ces juifs bien habillés deviennent des israélites ${ }^{8}$, elle, dans le même temps, se voit rebaptisée temple: Temple de la Victoire, Temple Notre-Dame de Nazareth, Temple des Tournelles. Le glissement lexical n'est jamais neutre.

Sans doute n'est-il pas inutile d'inverser l'ordre des termes pour poser maintenant cette autre question : quel lieu l'église est-elle, comparée à la synagogue ? Je serais tentée de suggérer que l'Église/ église se situe quelque part entre la synagogue et le Temple.

Sur le plan fonctionnel, en effet, l'église, comme la synagogue, est, à l'origine (c'est-à-dire avant que l'église ne devienne, dans l'Occident médiéval, le lieu fonctionnel disposant à l'accomplissement des sacrements $)^{9}$, une simple maison, une maison ouverte à tous, refuge pour les personnes recherchées, les enfants abandonnés, les sans-papiers. C'est la maison où les fidèles se rassemblent pour la prière, la lecture, l'homélie. En somme, comme la synagogue, c'est un « petit sanctuaire » accessible à tous.

7. Stéphane Mosès, Système et Révélation. La philosophie de Franz. Rosenzweig, Paris, Le Seuil, 1982 (préf. d'E. Lévinas), p. 30.

8. Simon Schwarzfuchs, Du Juif à l'israélite. Histoire d'une mutation (1770-1870), Paris, Fayard, 1989.

9. Voir ci-dessous la contribution de Dominique Iogna-Prat. 
Sur le plan spirituel et mystique cependant, c'est au Temple écrit avec un grand $\mathrm{T}$ que, peut-être, on pourrait se risquer à la comparer : à cet espace sacré, voué à Dieu et à Lui seul, où, séparé des fidèles et des simples humains, le prêtre consacré accomplit le sacrifice, dans la crainte et le tremblement. Pour pousser la comparaison, je serais tentée d'ajouter que depuis qu'il a été dépouillé de son enveloppe matérielle du fait de sa destruction, le Temple est devenu pure pensée pour les juifs, grâce au travail de la mémoire et de la tradition, «pensée du Temple » selon la belle expression empruntée à Francis Schmidt ${ }^{10}$, et, à ce titre, comme l'Église/corps mystique, objet d'adoration, voire d'idolâtrie mystique.

\section{LES AUTRES « LIEUX » EMBLÉMATIQUES DU JUDAÏSME DIASPORIQUE}

J'en viens maintenant à la question annoncée précédemment : existe-t-il, aujourd'hui, des «lieux » ou des types d'inscription spatiale dans lesquels les juifs de diaspora se reconnaissent de façon plus spécifique? Et si oui lesquels? Nous avons vu déjà, à propos d'Israël, que ces lieux sont divers et que tous n'ont pas nécessairement un caractère religieux, loin s'en faut. Quant aux « lieux » emblématiques du judaïsme diasporique, certains sont récents, nouvellement créés, d'autres anciens, recyclés ou affectés à de nouveaux usages.

Je commencerai par un bref inventaire des principaux lieux ou édifices à vocation juive, inaugurés, réalisés ou mis en chantier, au cours des dix dernières années: le Musée Mémorial de l'Holocauste de Washington, ouvert en 1993 dans la foulée d'une série de Monuments-Mémoriaux qui parsèment les États-Unis de Boston à San Francisco, le dernier en date étant le Musée de Battery Park construit à la pointe de Manhattan, face à la statue de la Liberté ; le Musée juif de Berlin, inauguré à la fin des années 1990 et, toujours à Berlin, le très controversé mémorial de la Shoah d'Eisenman; le Musée d'art et d'histoire du judaïsme de l'Hôtel de Saint-Aignan à Paris et, toujours à Paris, le Mur des Noms, récemment inauguré

10. Francis Schmidt, La pensée du Temple. De Jérusalem à Qoumran, Paris, Le Seuil, 1994. 
dans le Marais ${ }^{11}$. Là encore, cette liste n'est pas exhaustive. Dans cette liste, pas une seule synagogue ou lieu de prière. Ce n'est pourtant pas faute d'en avoir cherché.

Comment comprendre ou interpréter cet investissement massif dans la pierre, notamment dans la création de musées ? Doit-on y voir une marque d'assimilation culturelle, c'est-à-dire la preuve que les juifs auraient adopté les pratiques des gentils et qu'ils subiraient l'influence de la mode et d'un marché de la culture où la tendance serait en faveur des musées ? Faut-il y voir l'indice d'une certaine confiance dans l'avenir et la longue durée ? Ou faut-il y voir plutôt l'expression désabusée d'une volonté de laisser une trace de leur passage dans l'Histoire qui sache mieux résister aux flammes que les volumes de Talmud brûlés dans les autodafés de l'Inquisition ou que le bois des synagogues polonaises parties en fumée?

Preuve d'intégration et d'affirmation de soi ? Il ne fait pas de doute que les juifs se sont libéré d'une grande partie de leurs complexes. Ils se sont dépouillés de leurs habits de marranes, qui leur enjoignaient d'être «juifs chez eux, citoyens au-dehors ». Fini donc, pour beaucoup d'entre eux, le clivage schizophrénique qu'exigeait d'eux l'alignement sur le modèle dominant d'un Occident se confondant avec l'universel. Cela étant, désormais c'est dans la rue, bien plus que dans les synagogues que les juifs expriment avec le plus de force leurs angoisses. C'est collectivement et au grand jour qu'ils entendent faire connaître leurs convictions, autour de deux grands thèmes : le refus de l'antisémitisme et la solidarité avec Israël. Mais, ce n'est plus à Dieu que les «masses juives » adressent leurs prières et leurs cris de détresse ou de colère, c'est à la rue, aux médias, à leurs frères humains. Il n'est pas indifférent de rappeler que, bien avant les grandes manifestations de Paris de l'après Carpentras (profanation du cimetière juif en 1990) ou d'avril 2002, ce sont les juifs de Moscou qui, les premiers, dans les années 1960-1970, ont donné le ton, en pleine période de répression,

11. Remarquons que l'inscription de lettres sur la pierre n'est pas une nouveauté pour les juifs. Cela les renvoie aux origines mêmes de leur tradition, au Sinaï et aux tables de la Loi. À cette différence près que les tables de la Loi étaient supposées être la transcription de la Parole de Dieu, alors que les inscriptions actuelles portent les noms des victimes de la Shoah. 
avant même que les premiers refuzniks juifs n'obtiennent leur visa de sortie. Chaque année, alors, à l'occasion de la fête juive de Sim 'hat Torah, de jeunes juifs moscovites, de plus en plus nombreux d'année en année, avaient pris l'habitude de se rassembler dans la rue Arkhipova, devant la grande synagogue de Moscou, et ce malgré la présence des caméras du KGB. Ils avaient inventé cette forme de manifestation pour exprimer, en tant que juifs, l'éveil de leur nouvelle conscience collective. Pourquoi devant la synagogue ? Parce qu'elle était un symbole, un symbole identitaire, unitaire, un symbole d'appartenance, le seul disponible. Mais alors pourquoi pas dans la synagogue ? Précisément parce qu'elle n'était qu'un symbole et rien d'autre. Il faut savoir en effet que, pendant la manifestation qui se déroulait dans la rue, la synagogue, elle, était presque vide. $\mathrm{Ne}$ s'y trouvaient que quelques vieux, des habitués. Les jeunes, eux, n'y entraient pas. D'ailleurs, ils ne savaient pas prier et cela ne les intéressait pas. Ce qui était important à leurs yeux, alors, c'était le fait d'être ensemble à l'extérieur et de s'y compter.

La synagogue, symbole unitaire, certes, mais aussi expression de la diversité, voire de la fragmentation, de la conflictualité et qu'illustre ce dicton juif bien connu selon lequel tout juif a besoin de deux synagogues : celle où il va et celle où il ne va pas. Aussi et tout compte fait, l'application aux juifs de la démarche sociologique Le Brasienne ne serait pas si absurde que cela. L'étude sociologique de la fréquentation de la synagogue ou de la petite salle de prière de quartier, qu'elle se trouve à Jérusalem, à Sarcelles ou dans un shtetl de la Pologne d'avant-guerre, peu importe, pourrait s'avérer un révélateur précieux de cette diversité, et pas seulement des clivages entre pratiquants, réguliers, occasionnels ou festifs.

En effet, une sociologie, non pas religieuse mais politique, de la synagogue reste à faire. Car le rite, l'origine (séfarade/achkenaze), l'obédience religieuse (orthodoxe, traditionaliste, réformée ; hassidim/ mitnagdim $)^{12}$ sont loin d'être les seuls indicateurs pertinents de la

12. Les hassidim d'aujourd'hui sont les héritiers d'un mouvement populaire piétiste né en Podolie au XVIII ${ }^{\mathrm{e}}$ siècle et dont le maître à penser est passé à la postérité sous le nom de Baal Chem Tov (le Maître du Bon Nom). Les Mitnagdim (opposants) étaient les adversaires de ce mouvement. 
diversité juive. Pour preuve, l'histoire suivante, véridique. L'action se situe il y a un peu plus d'un siècle, autour des années 1890-1900, dans une petite ville de Pologne, à quelques dizaines de kilomètres de Lodz, un centre important de l'industrie textile. Une grande partie de la population juive du lieu est employée dans l'industrie textile. D'un côté, il y a ceux qu'on appelle « les gros », les employeurs, les entrepreneurs, les patrons de manufactures, les intermédiaires intéressés au chiffre d'affaire ; de l'autre, « les petits », petits patrons qui pratiquent la sous-traitance à domicile, qui travaillent avec femmes, enfants et apprentis, parfois avec un ou deux salariés, et les ouvriers, ceux qui travaillent dans les manufactures. Depuis toujours, dans le climat paternaliste qui caractérise le petit monde du shtetl, patrons et ouvriers se retrouvent le chabbat et les jours de fêtes à la synagogue : les premiers aux places d'honneur, les seconds entassés sur les bancs du fond. Jusqu'au jour où la politique et la conscience de classe font brusquement irruption dans ce petit monde qui vit encore au rythme de la tradition et de la prière. À partir de ce jour, les « petits » ne supportent plus l'idée de prier aux côtés des « gros », leurs exploiteurs. C'est pourquoi ils décident de manifester leur refus en fondant un syndicat, mais aussi en créant leur propre oratoire pour y prier «entre eux ». Ainsi pour ces juifs-là, c'est dans le lieu de prière lui-même que commence la lutte des classes. Aujourd'hui encore, les synagogues de diaspora sont loin d'être des lieux politiquement neutres. Elles sont un des lieux d'expression naturelle de la solidarité des juifs avec Israël, et certaines en paient le prix en devenant les cibles d'agressions antisémites ou antisionistes, tant il est difficile, parfois, de faire la différence.

Enfin, et ce sera mon dernier point, que dire de cette nouvelle « religion pèlerine », pour reprendre la formule de Danièle HervieuLéger $^{13}$, que constitue le tourisme de mémoire? Que dire de ces visites organisées sur les lieux de la vie juive d'antan et de ses vestiges architecturaux ? Que dire de ces synagogues historiques, à Prague ou à Cracovie, dont la restauration vise moins à les rendre à leur vocation première qu'à les transformer, au mieux en musées-

13. Danièle Hervieu-Léger, La religion en mouvement. Le pèlerin et le converti, Paris, Flammarion, 1999. 
lieux de mémoire, au pire en produits touristiques ? Que dire du Mur occidental et des foules de fidèles qui s'y pressent ? Source de confusion extrême des genres et des esprits, cet unique vestige du Temple est devenu une immense synagogue à ciel ouvert où des juifs s'adonnent sans retenue à un rite païen et idolâtre d'adoration de la pierre et du lieu. Que dire enfin de ces nouveaux pèlerinages sur les lieux de la mise à mort industrielle des juifs, de ces visites guidées, souvent conduites ou commentées par d'anciens déportés, dans les camps d'extermination? Autant que le Temple qui faisait l'admiration de Flavius Josèphe et dont la mémoire a été pieusement conservée par la tradition et le rite, autant que la synagogue de type traditionnel ou que la yechiva qui attire aujourd'hui tant de jeunes déboussolés, en quête de certitude, d'absolu, de réinscription dans une lignée croyante, autant que les monuments et les musées aux architectures imposantes, ce sont, là aussi, les nouveaux lieux emblématiques du judaïsme contemporain; des lieux où s'expérimente une modalité, jusque-là inédite chez les juifs, du rapport au passé, en même temps qu'une modalité du croire qui n'est plus celle de la tradition instituée. Ceux, juifs et non-juifs, qui se rendent sur ces lieux de désolation et de mémoire, ces lieux qui attestent de la faillite d'une civilisation, nourrissent souvent l'espoir qu'en s'y rendant, ils y trouveront une forme de réparation ou qu'ils pourront y renouer le lien rompu avec leurs origines.

azria@ehess.fr 